\title{
Current issues on HIV adults in South East of Romania
}

\author{
Manuela Arbune ${ }^{1,2}$, Mihaela Debita ${ }^{1,2}$ \\ ${ }^{1}$ Infectious Diseases Clinical Hospital, Galati, Romania \\ ${ }^{2}$ Medicine and Pharmacy Faculty, "Dunarea de Jos" University, Galati, Romania
}

\begin{abstract}
Objective. To identify the current priorities of the fight against HIVIAIDS in the South-East region of Romania. Methods. The profile of HIV adult confirmed with HIV, the mortality rate and the risk factors related to death were analyzed in a retrospective study.

Data were collected from the medical files of Galati HIVIAIDS Clinic. New diagnosed HIV infected patients, over age 15 were followed-up from January 2005 to December 2016.

Results. During the 12 years study period, 225 new HIV adult cases were confirmed. The characteristics of the new diagnosed cases were: young age (average $25.17 \pm 11.94$ ), predominant heterosexual transmission mode $(73 \%)$ and high percentage of late presentation $(67.6 \%)$. The retention in care rate was $91 \%$ and $95 \%$ of patients received ART. The mean annual mortality rate was $14.6 \%$, one third of deaths occurring during the first year after diagnosis. In the last 6 years, there was an increase trend in mortality rates, compared to the period 2005-2011, as HIV people are aging and more non-AIDS deaths are registered. The survival rates were not influenced by the first line ART, containing protease inhibitors, non-nucleoside reverse transcriptase or integrase inhibitors. However, all ART regimen significantly improved the survival rate.

Conclusions. Even if ART improved HIV survival rates, the mortality rate among persons diagnosed with HIV was still higher than in the general population. As HIV patients are aging, continuing increase in non-AIDS comorbidities are expected in the next years. The local present priorities in the fight against HIV are to enhance the efficiency of ART, early HIV diagnosis and retention in care.
\end{abstract}

Keywords: HIV, non-AIDS diseases, late presenter, mortality

\section{BACKGROUND}

Since the beginning of the HIV epidemic, an estimated 76.1 million (65.2 million-88.0 million) people have become infected with human immunodeficiency virus (HIV) and 35.0 million (28.9 million-41.5 million) people have died because of related illnesses of acquired immunodeficiency syndrome (AIDS). In 2016, around 53\% (39-65\%) of people living with HIV from all over the world had access to antiretroviral treatment (ART) (1). ART has considerably increased the life expectancy and the quality of life among people with HIV. The mortality rates in persons living with HIV who receive ART is close to the mortality in general population $(2,3)$. Therefore, the approach of HIV infection changed, from a fatal to a chronic disease. The mortality rates among people with HIV are different around the world, depending on the economic rank, the accessibility to ART, the regional health strategies for HIV prevention and treatment, and also on general factors in the general population, as behaviors, environment and genetic factors.

The European Centre for Disease Prevention and Control (ECDC) supports the actions in HIV continuum of care and monitors the response to the HIV epidemic in European countries4. As an European country, Romania is adhering to the "90-9090" UNAIDS target for continuous care. This suppose that by $2020,90 \%$ of all people living with HIV will know their HIV status, $90 \%$ of all people 
with diagnosed HIV infection will receive sustained antiretroviral therapy and $90 \%$ of all people receiving antiretroviral therapy will have viral suppression5. Sustained viral suppression has long term benefits for the each patient, due to the improvement in survival and quality of life and also for the public health due to reduced viral transmission in community $(6,7)$.

The aim of our study was to evaluate the current characteristics of Romanian adults diagnosed with HIV, the mortality rate and the risk factors of death, in the era of effective antiretroviral treatment.

\section{MATERIAL AND METHODS}

Retrospective study on HIV infected patients, followed- up in Infectious Diseases Clinical Hospital Galati, between 1st of January 2005 and 31st of December 2016. Demographic characteristics, mortality rate and risk factors of death were assessed for adults diagnosed with HIV. The demographic data, risk factors for HIV infection, HIV related co-morbidities, baseline CD4 cell counts (LCD4) and type of first line ART, were collected according to the special forms for case notification developed by Romanian National Committee against HIV/AIDS. Criteria for "late presenter" was considered LCD4 under $350 / \mathrm{mm} 3$ or the evidence of an AIDS condition.

Inclusion criteria was the age of patients over 15 years old. The causes of deaths were divided in AIDS and non-AIDS related-deaths. The retention in care rate, the mortality rate and the causes of deaths were evaluated. The retention in care rate was calculated as the percentage between the number of patients with continuous follow-up during 12-months and number of patients at the study initiation. Kaplan Meier plots estimated the survival according to the type of the first line ART, compared with no treatment. The ART regimens were containing one of the following: protease inhibitors (PIs), non-nucleoside reverse transcriptase inhibitors (NNRTIs) or integrase inhibitors (INSTI).

Statistical analysis was performed using descriptive and analytic methods, including logistic regression and correlation by Pearson $\chi 2$ test. Statistical significance was considered at a $p$ value $<0.05$. The analysis was performed with XL-STAT software.

\section{RESULTS}

From 2005 to 2016,423 HIV infected patients over age 15 were recorded for medical procedures in Infectious Diseases Hospital Galati. A number of 225 patients with mean age $29.88 \pm 12.15$ were newly diagnosed during this time (Fig. 1). The overall cascade of care included $8.8 \%$ of patients lost from evidence and $14.1 \%$ death. Noteworthy, one third of deaths occurred in the first year after HIV diagnostic.

All the patients were of Caucasian race. The main characteristics of the new HIV cases were: age under 30, predominantly male, smoker, late presenter and with ART started in the first year of HIV diagnosis (Table 1).
FIGURE 1. Annual evolution of cases in Galati Clinic (1989-2016)

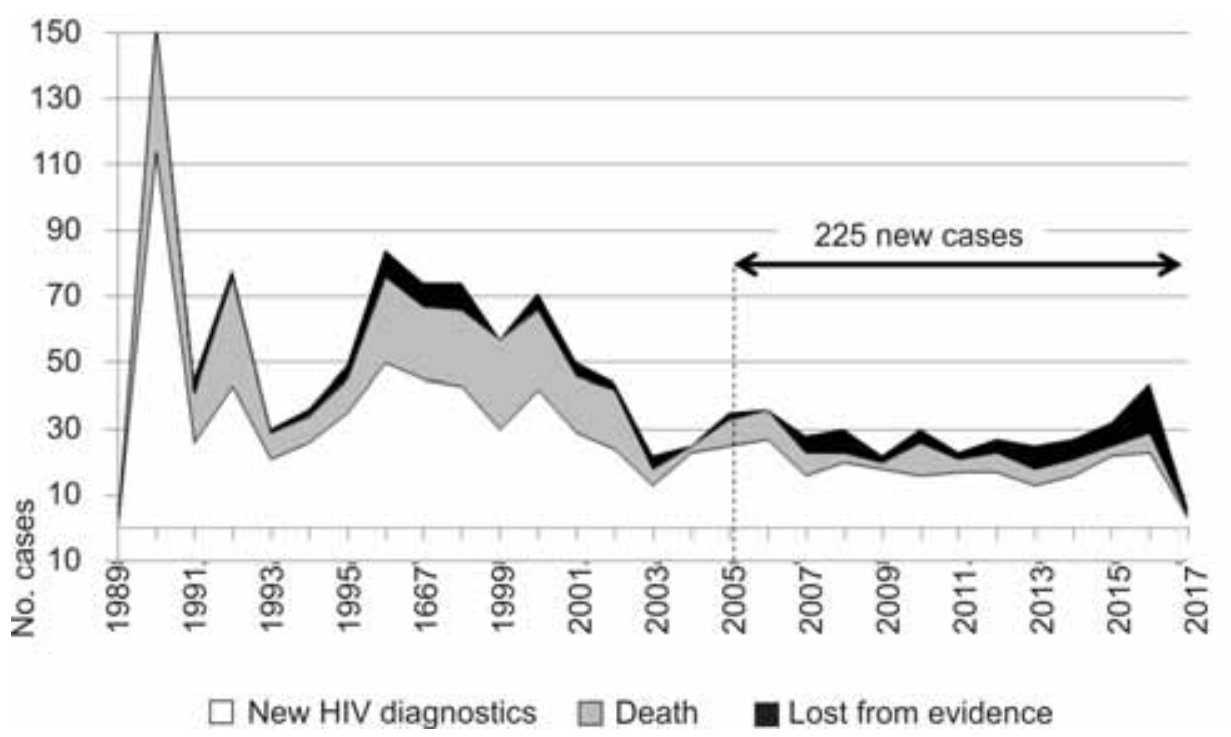


TABLE 1. Characteristics of the new cases diagnosed with HIV between 2005 and 2016

\begin{tabular}{|l|c|c|c|c|c|}
\hline Categories & Yes & No & $\%$ & Cl 95\% & P value \\
\hline Age at diagnosis < 30 years & 120 & 85 & $60 \%$ & $0.54 ; 0.67$ & 0.001 \\
\hline Gender (male) & 126 & 99 & $56 \%$ & $0.49 ; 0.62$ & 0.071 \\
\hline Living in urban area & 114 & 111 & $51 \%$ & $0.44 ; 0.57$ & 0.841 \\
\hline Smoker & 139 & 86 & $62 \%$ & $0.55 ; 0.68$ & 0.001 \\
\hline $\begin{array}{l}\text { Route of HIV acquisition } \\
\text { heterosexual }\end{array}$ & 164 & 61 & $73 \%$ & $0.67 ; 0.78$ & $<0.001$ \\
\hline History of temporary migration & 73 & 152 & $32.4 \%$ & $0.26 ; 0.38$ & $<0.001$ \\
\hline History of incarceration & 30 & 195 & $13.3 \%$ & $0.08 ; 0.11$ & $<0.001$ \\
\hline Hepatitis B co-infection & 39 & 186 & $17.3 \%$ & $0.12 ; 0.22$ & $<0.001$ \\
\hline Hepatitis C co-infection & 15 & 210 & $6.6 \%$ & $0.03 ; 0.09$ & $<0.001$ \\
\hline Late presenter (CD4<350/mm ${ }^{3}$ ) & 152 & 73 & $67.6 \%$ & $0.61 ; 0.73$ & $<0.001$ \\
\hline On antiretroviral treatment & 202 & 23 & $90.0 \%$ & $0.85 ; 0.93$ & $<0.001$ \\
\hline
\end{tabular}

The main HIV transmission route was heterosexual $(73.0 \%)$, following by nosocomial route (16.0\%), injecting drugs use $(1.77 \%)$ and sex between men $(1.33 \%)$ and unknown in $8 \%$ of cases. One third of new diagnosed HIV patients experienced temporary migration and $19 \%$ had a history of incarceration.

The leading circumstances of HIV diagnosis were the recommendation for symptomatic patients with fever, diarrhea, candidasis, adenopathy and weight loss (Fig. 2).

The most frequent co-morbidities among new HIV cases were cachexia (30\%), tuberculosis $(29 \%)$ and shingles (29\%). Co-infection with hepatitis $\mathrm{B}$ virus was detected in $22 \%$ and with hepatitis $\mathrm{C}$ in $6 \%$ of cases. The most frequent sexually transmitted co-infections were: genital condyloma $(10 \%)$ and syphilis (9\%). Less frequent complica- tions were: cerebral toxoplasmosis $(3 \%)$, lymphoma $(2 \%)$, cryptococcal meningitis $(2 \%)$ and Pneumocystis jirovecii pneumonia (1\%).

There were 29 deaths among HIV infected patients. The annual mortality rate range between 26.4/1000 (2006) and 0 in 2007 and 2008. The mean HIV annually mortality rate was 14.6 $\pm 8.3 / 1000$ HIV people, being 1.4 fold higher than the regional mortality in the general population in the same period (Fig. 3).

The main causes of death were: tuberculosis, non-Hodgkin lymphoma and Kaposi sarcoma. We noticed an increase in non-AIDS related diseases staring with year 2011 (Fig. 4).

The median age of death with non-AIDS related diseases was higher than the age of death related to AIDS-disease (Fig. 5).

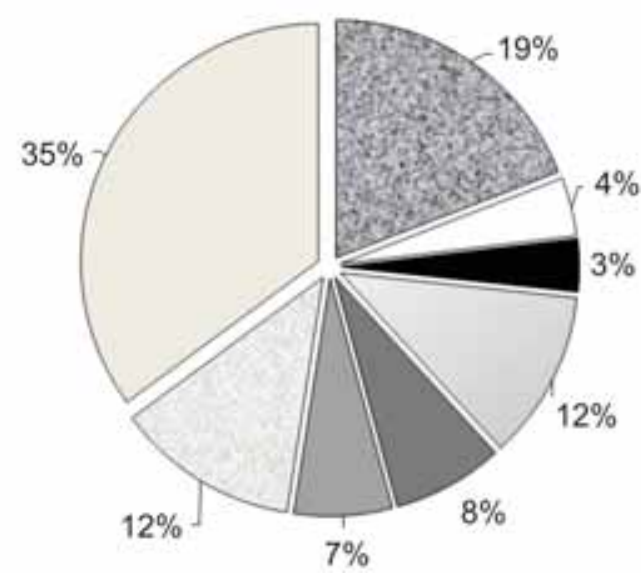

v contact of HIV + partner

$\square$ voluntar test

- blood donnor

口pregnancy

vsexual transmitted diseases

घother screening programmes

atuberculosis

पrecommended to symptomatic patients

FIGURE 2. Circumstances of HIV diagnostic in adult patients (2005-2016) 


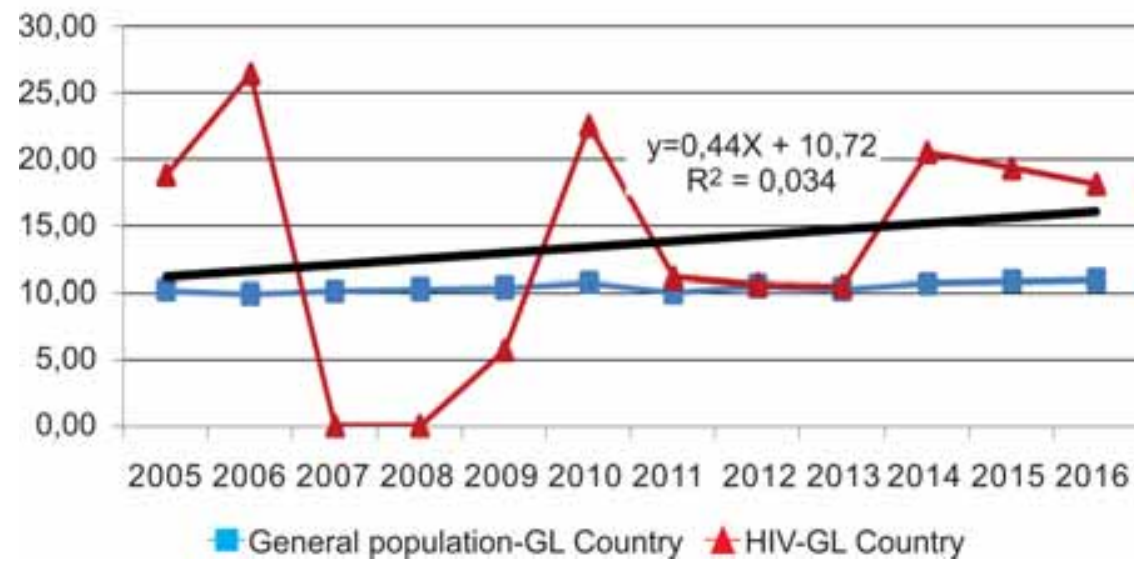

FIGURE 3. Evolution of mortality among HIV patients and general population from Galati County

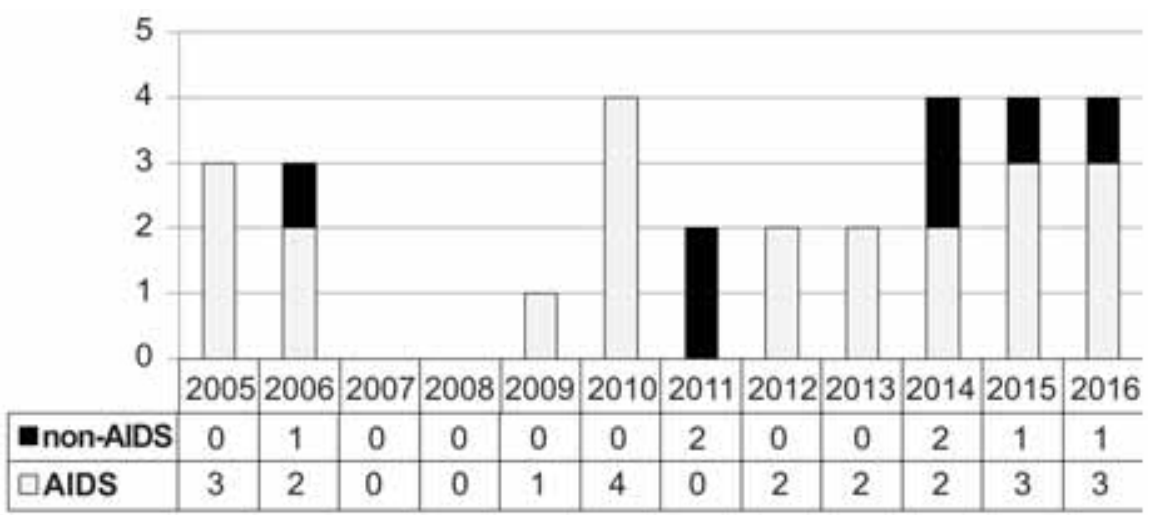

FIGURE 4. AIDS and non-AIDS related deaths (2005-2016)

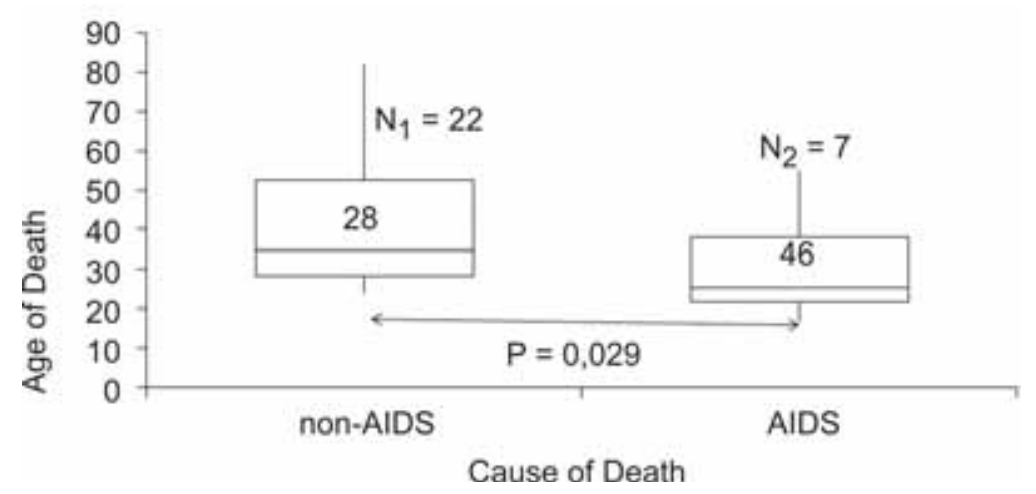

FIGURE 5. Comparative median age of death related to AIDS and non-AIDS conditions

The ART was commonly available for all HIV infected patients. The first line of antiretroviral therapy was based on protease inhibitors (PIs) $52.6 \%$, non-nucleoside reverstrascriptase inhibitors (NNRTIs) $39.4 \%$ or integrase inhibitors (INSTI) $7.9 \%$. There was no influence on survival depending on the type of ART. However, Kaplan Meier plots, showed a longer survival in patients on ART compared to those without treatment (Fig. 6).
The durability of first line ART was sustained only in $38 \%$ cases. The other outcomes were: $18 \%$ abandoned ART, 24\% failed ART and 20\% changed the treatment because of adverse reactions or drugdrug interactions. HIV viral suppression was achieved in $68 \%$ of patients on ART.

The occurrence of death was influenced by smoking, late presentation, tuberculosis co-infection and absence of antiretroviral treatment (Table 2). 


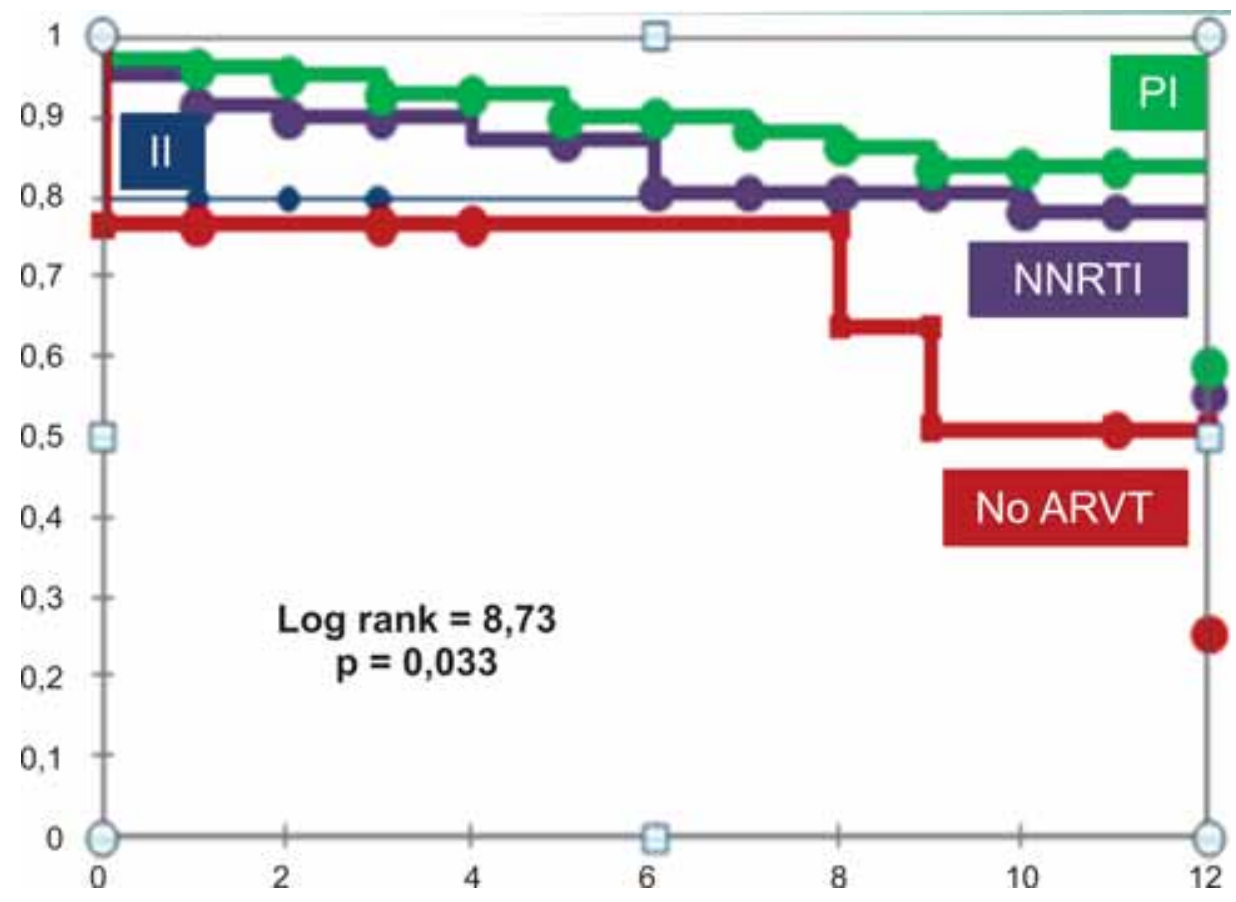

FIGURE 6. Comparative survival distribution depending on first line ART based on PIs, NNRTIS, INSTI or No-ART

We didn't observe any significant correlations of mortality with neuro-toxoplasmosis, sexually transmitted infections or hepatitis B and C.

TABLE 2. Correlations of risk factors for death among HIV patients (2005-2016)

\begin{tabular}{|l|c|c|c|}
\hline Risk factors & OR & Cl 95\% & p value \\
\hline Male & 2.29 & $1.1 ; 4.4$ & 0.015 \\
\hline Smoking & 3.82 & $1.4 ; 9.9$ & 0.005 \\
\hline Tuberculosis & 3.07 & $1.4 ; 6.7$ & 0.004 \\
\hline Late presenter & 4.48 & $1.4 ; 14.8$ & 0.010 \\
\hline No-ARVT & 3.45 & $1.1 ; 10.3$ & 0.026 \\
\hline
\end{tabular}

\section{DISCUSSIONS}

The Romanian HIV epidemic is particular due to a high number of cases belonging to the cohort infected between 1987 and 1990, known as the "Romanian pediatric HIV cohort". Three decades ago, an incomplete clarified epidemiological event occurred and many small children become HIV infected, probably after blood transfusions, parenteral treatments, hospitalizations or institutional care, although their mothers were HIV negative. At the begin of 1990, HIV testing became accessible in Galati County, as well as in other regions of the country, and increasing number of HIV pediatric cases (100) fueled the new epidemic. During the time, a part of the patients from the pediatric cohort died and the remaining grew up and become adults. A second pattern of the HIV epidemic was developed concomitant with the pediatric epidemic, involving adults infected by heterosexual mode.

In the recent years, the pattern of HIV epidemic is changing and new cases are more frequent heterosexually infected adults. A percent of $16 \%$ long term survivors from the pediatric cohort were diagnosed during the first interval of the study, but not in the last years. We registered HIV infections among MSM and intravenous drug users, although these transmission routes are still rare in our region.

The proportion of new HIV cases in females is higher compared with most EU countries, where transmission by sex between men is commonly reported (8).

Prisons are vulnerable groups with high risk of HIV acquisition, frequently associated with unhealthy behaviors, as drug use, multiple sexual partners, or smoking. One third of HIV new cases from Galati had a history of working abroad or temporary migration before HIV diagnosis. These are to date important social and demographic problems in the general population in all over the country. Migration and HIV is a complex issue across Europe, mostly related to the integration of foreign- 
ers with HIV and to supply the health care resources. In 2015, the proportion of migrants among newly-diagnosed HIV cases in European Union countries was variable, ranging from over $70 \%$ to less than 5\% (9). Immigration in Romania is less significant until now, even in the refugee centers, as Galati. Following the HIV screening of refugees, only 3 males were found positive and all they leaved the centre in few weeks after diagnosis. On the other side, emigration of Romanian HIV patients is the main risk for retention in care in our site and become a problem of immigration in other European countries.

The rate of late presenters was higher than the average rate across EU (51\%) and even compared to the national data $(67.7 \%$ vs. $64.0 \%) 8$. Late presentation in our study was significantly correlated with male gender and the risk of death (Table 2).

Over one third of patients were symptomatic at the time of diagnosis, being in concordance with the high proportion of the reported late presenters. Tuberculosis was the most frequent opportunistic infection and reason for disclosure the HIV diagnostic.
In concordance with the WHO's recommendations to initiate ART in all people with HIV, over $90 \%$ of patients from Galati are retained in care and $90 \%$ received at least one line of medication (10). Although this objectives of HIV control strategy were reached, the results of the treatment are less optimistic, due to the low rate of HIV viral suppression.

\section{CONCLUSIONS}

The HIV epidemic in the South East of Romania is relatively stable, regarding the number of new cases. Antiretroviral treatment improved the life expectancy in people living with HIV, although the mortality is still higher than in the general population. AIDS-related diseases are still the majority cause of HIV related deaths. However, non AIDS co-morbidities tend to increase and are expected to be prevalent in the next years, as HIV survivors are aging. The objectives of our local present strategy of fight against HIV are to enhance the efficiency of ART, but also to encourage the early HIV diagnosis and the retention in care.

\section{REFERENCES}

1. Joint United Nations Programme on HIVIAIDS (UNAIDS). [Internet] UNAIDS Data 2017 [cited 02.03.2018] Available from: http://www. unaids.org/sites/default/files/media_asset/20170720_Data_ book_2017_en.pdf.

2. Bhaskaran K., Hamouda O., Sannes M. et al. Changes in the risk of death after HIV seroconversion compared with mortality in the general population. JAMA, 2008; 300: 51-59.

3. Simmons R., Ciancio B., Kall M., Rice B., Delpech V. Ten-year mortality trends among persons diagnosed with HIV infection in England and Wales in the era of antiretroviral therapy: AIDS remains a silent killer. HIV Med, 2013; 14: 596-604.

4. Drew R.S., Rice B., Rüütel K., Delpech V. et al. HIV continuum of care in Europe and Central Asia. HIV Medicine, 2017;18:490--499.

5. UNAIDS. 90-90-90 An ambitious treatment target to help end the AIDS epidemic [Internet]. Geneva; 2014 [cited 2016 Aug 29]. Available at: http://www.unaids.org/sites/default/f iles/media_ asset/90-90-90_en_0.pdf.

6. Loutfy M.R., Wu W., Letchumanan M. et al. Systematic review of HIV transmission between heterosexual serodiscordant couples

where the HIV-positive partner is fully suppressed on antiretroviral therapy. PLoS ONE, 2013; 8(2):e55747.

7. Cohen M.S., Chen Y.Q., McCauley M. et al. Prevention of HIV-1 infection with early antiretroviral therapy. N Engl J Med 2011; 365(6):493-505.

8. European Centre for Disease Prevention and Control/WHO Regional Office for Europe. HIVIAIDS surveillance in Europe 2017 - 2016 data. Stockholm: ECDC; 2017.

9. European Centre for Disease Prevention and Control. HIV and migrants. Monitoring implementation of the Dublin Declaration on Partnership to Fight HIVIAIDS in Europe and Central Asia: 2017 progress report Stockholm: ECDC; 2017.

10. World Health Organization. Consolidated guidelines on the use of antiretroviral drugs for treating and preventing HIV infection. Recommendations for a public health approach. Second edition. Geneva: WHO; 2016. Available at: http://apps.who.int/iris/bitstr eam/1 0665/208825/1/9789241549684_eng.pdf?ua=1. 